\title{
Practical Dilemma and Elaboration of MOOC out of The Existence of Internet
}

\author{
Jing Zhang \\ School of Public Affair and Law \\ Southwest Jiaotong University \\ Chengdu, Sichuan, China,
}

610031

\author{
Liyun $\mathrm{Lu}$ \\ School of Public Affair and Law \\ Southwest Jiaotong University \\ Chengdu, Sichuan, China,
}

610031

\begin{abstract}
As an innovative way of teaching and learning, MOOC has impacted the traditional teaching mode. By analyzing data and consulting literature, this paper points out the present situation of Chinese MOOC. On the bas is of investigations, this paper lists several problems exis ting in the practical development of MOOC including multiple platforms, low completion rate and the rising dropout rate, poor quality of courses and the lacks of characteristics, the limited source of funding as well as the weak authentication system. To solve these problems, this paper provides five summarized ways. Construct a unified platform, to improve the quality of courses on multiple aspects, to produce distinguished courses, to explore the commercially operational modal and set up thoroughly developed authentication system. These five paths of problem-solving have important reference value for the reform and prosperity of Chinese MOOC education.
\end{abstract}

Keywords_Information Technology; MOOC; Dilemma; Path

The $21^{\mathrm{s}}$ century is a period of rapid development of science and technology. The prodigious usage of network has greatly changed the way of production and people's lifestyles. The combination between enormous Internet data and traditional education has created a new education mode: "MOOC". "MOOC"- massive open online courses-. "Mass" is the colossal number of people involved in the processes of studying; "Open" refers to, without strict and explicit restrictions, open access to participants from all over the world; "Online" is intended to be published on the online platforms. Enrolled users participate in online interaction and communicate with tutors via the platforms; "Courses" reckons that the various courses offered on MOOC platforms are from best universities in the world. While offering low-cost and even free advanced education services to users, MOOC integrates online learning, online communication feedback, data integration and analysis. As a new configuration of education, MOOC received extensive attention from the society within the short term and reached its prosperity. However, after attentiongrabbing, rapid expansion of MOOC possesses itself with greater friction that hindrance appeared. Exploring how to solve these contradictions has become a hot topic in the current educational research.

This work is supported by "the Fundamental Research Funds for the Central Universities" --The realistic factors that affect the judicial identification of the public and its countermeasures.

\section{ORigin ANd Development of MOOC Education}

Early in 2003, American scholar M ahnaz M oallem proposed the significance of course learning about multimedia and network communication tools for online classes[1]. The term MOOC firstly appeared in 2008. Professor George Siemens, a researcher at the national research council, who is from the University of Athabasca and Stephen Downes, opened a course called " Connectivity and Connective Knowledge" for 25 tuition-paying students at the University of Manitoba in Extended Education, and linked access to enrolled users around the world without charges. However, more than 2200 students eventually took part in the course. For the first time, Professor Dave Cormier from Prince Edward Island University put forward the concept of "MOOC". In 2011, some professors from Stanford University had sequentially released videos which were recorded and played through online platforms. Sebastian Thrun and professor Peter Norvig jointly opened a free online course called 'Introduction into AI", which attracted students from all over the world with rapid enrollment of 160,000 students. In this case, professor Sebastian Thrun founded Udacity, a private education agency. Later, professors Daphne Koller and Andrew Ng from Stanford university co-founded Coursera, a company that aims to launch free online courses with the top universities in the world. Coursera then announced a partnership with the university of Pennsylvania, Princeton, Stanford, and the University of Michigan. In the same year, MIT and Harvard University jointly developed the nonprofit Edx online learning platform. Since then, the three substantial suppliers of MOOCs were established. Thus, the New York Times called the year of 2012 'the first year of MOOC" as MOOCs spreading around the world.

China joined the trend of the development of MOOCs comparatively early. In 2013, China has begun to introduce MOOCs and constructed the very their own platforms. Peking University and Tsinghua University have reached a partnership with the Edx platform in May 2013; Fudan University has signed a letter of intent with Coursera; Shanghai Jiaotong University has started to offer courses to Coursera in July 2013. The world's first Chinese MOOC platform, built by Tsinghua university, has been officially become serviceable. Various courses from Chinese universities were uploaded and offered to the public in 
October 2013. Chinese MOOC has developed from scratch on the basis of reference and innovation.

\section{The PROBlems EXISTING IN THE EMPIRICAL DEVELOPMENT OF MOOC}

2013 is called the first year of Chinese MOOCs as many colleges and universities were actively involved in the construction of MOOCs and the greater attention was paid to MOOCs. However, with the rapid development of local MOOCs, potential problems have been highlighted and became seriously. Main performances are:

\section{A. Multiple platforms that lack of unified resources and promotion}

From the government-founded platforms to the three world famous MOOC providers "Coursera", "Udacity" and "Edx", they all have relatively complete curriculum system, enormous number of registered users, aiming to form an inter-school cooperation alliance, and build a unified sharing platform. On the view of Chinese indigenous MOOC platforms, there are no unified rules and regulations, and there are sundry MOOC platforms in China, including educational company, which is represented by "school online". There are colleges and universities represented by "good university online"; there is also a combination of education publishing company and Internet company, represented by "MOOC of Chinese University". As is known to all, the production of MOOCs and the operation of the platform are not easy. The release of information, data collection, integration analysis and daily maintenance of the platforms all require strong financial support and technical support. However, the multitude of MOOCs can easily lead to low utilization of the platform and the construction of repetitive labor, resulting in the waste of resources. At the same time, the team which makes MOOCs is lack of inner communication, which tends to reduce the efficiency. In addition, the unified platform also makes learning more difficult for enrolled users and dampens their enthusiasm for learning.

In addition, the foreign MOOCs model, which initially did not provide credit certification, is still highly regarded, and has developed rapidly in a few years. This is due to the early start of developed countries, and the Internet learning style, which has become more common and popular. On the other hand, the promotion of commercial publicity is more powerful, attracting more enrolled users in the short term to join the new learning mode of MOOCs. For example, Edx actively seeks cooperation with Facebook, Twitter and other social networking platforms to promote business and expand its influence. On the view of China, although universities are actively building MOOC platforms and making courses of high quality, because the platform relies on the universities and the government, the lack of commercial elements, the lack of systematic marketing and promotion, the results are little known.

In our opinion, MOOCs are designed to expand the scope of education radiation, and to make the remote areas feel the benefits of high-quality teaching resources. In fact, due to the lack of publicity and promotion, there are fewer opportunities for the general public to contact with the MOOC. The MOOC mode is only circulated among college students. If the general public know little or nothing about MOOC model, the socalled free popularization of education and institutional change will also be an empty talk.

\section{B. Low completion rate and the elevated dropout rate}

A study from the United States in 2013 "MOOC and open education, the meaning of higher education" reveals that, according to University of Stanford, MIT, university of California, Berkeley, dropout rate of MOOC ranges from eighty-five percent to ninety percent. Moreover, graduate rate of registered students on the Udacity platform is merely about 10 percent [2]. Although MOOCs have numerous enrolled students, only few of them eventually have the courses completed. For example, one of the Shanghai Jiaotong University's first online courses, "Journey of mathematics", is scarcely completed with three percent overall finishing rate [3] Besides, the course, named 'how to create MOOC", targeting to Chinese university students, has initially a total enrollment of 15,758 students, but only 806 students completed tasks within the course [4]. Multiple reasons existed for explaining such phenomenon, ignoring the objective situation of the course, that participants have no motivation and ability to finish these courses can be the uppermost factors, which causes such harshness of MOOC. On one hand, the openness of the MOOCs inevitably leads to the randomness of the learning process that students may enroll in MOOCs out of curiosity, but it is not motivated enough for them to prudently enroll and completely finish the courses. Additionally, tuitionfree courses, without penalty when dropping courses on the purpose of promoting MOOC, are easily dropped by students after fervor dims. On the other hand, though MOOCs are characterized with high interactivity and establishes a lot of reciprocal BBS, students often fail to get answer and feedback online punctually, resulting in low learning efficiency and ultimately having difficulty in completing the course.

\section{Courses' quality is uneven and lacks of characteristics}

Many courses do not teach knowledge practically to students but just simply provide video-academy in which the knowledge offered is rigid, or the duration of classes is too long and can't be defined as MOOCs. It is difficult for enrolled users to use fragmented time to get partial learning and leads to low-efficient learning. On the other hand, gap exists in the courses both in the top-ranked Chinese and foreign universities that local MOOCs are neglected when others are fully registered. Meanwhile, majority of the best MOOCs are provided by well-known universities in the western developed countries and English-based. With only few subtitles in Chinese, it indirectly limits the number of enrolled users. Into the bargain, the courses of western universities are often combined with their own practical and cultural features. So, some of them feel difficult to understand the courses. However, the online courses produced by domestic universities are mostly duplicated from foreign platforms in terms of performance and function. Ignoring the application of Chinese culture, the quality of lectures can be 
inferior and lacks individuality as well while resulting in overall substandard quality of the courses.

\section{The source of funding is limited and lack of commercially operational system}

The establishment and maintenance of MOOC platform, the updating of courses, and the development of interactive technology require financial support. Only a large amount of investment funded can promote the daily operation of a platform. The construction of MOOC in Europe and America is mainly driven by Internet capital and venture capital. Coursera and Udacity in the MOOCs have established a forprofit platform at the beginning of their establishment and can finance themselves by offering high-quality courses and certificates. Edx is a not-for-profit platform, but it receives more than ten million dollars from all sectors of society [5]. Foreign MOOC financing channels are diversified. In addition to government financial allocation, there is a large influx of social capital, such as corporate sponsorship and social donation. The MOOC construction in European and American colleges and universities is a combination of school and enterprise, the research and production of the courses, and the commercial promotion of the courses. In China, there is more cooperation between universities and government. Due to the relatively lagging development of the foundation in China, the investment of social capital is less, and the fund of MOOC platform is mainly derived from government financial allocation. In addition to the construction of the university, the university has received the educational funding from the government. Besides, it also needs to be used for the maintenance of the MOOC platforms, being unable to deal with a situation. The operation of the MOOCs that relies on the input of government and university may bring a boom in the short term, but it is actually not conducive to long-term and sustainable development.

\section{E. The authentication system is weak}

In the aspect of the recognition of MOOC's credit, some countries in the world have been exploring and studying for a long time. Such as the UK higher education credit accumulation and transformation system and European credit swaps and accumulation system, South Korea's "credit bank" system and promotions of all kinds of education building in Australia authentication framework, etc. Some universities in China are also actively preparing for the credit certification. Wuhan Bioengineering Institute, for example, admits that the credits earned by students at the "Chinese university MOOC" have the same effect with the school's public elective courses. Peking university admits the students' credit for the courses offered by teachers of their own. And a course called "Mechanical Design" of Southwest Jiaotong university will enable enrolled users to complete the course certificate issued by the professor. In spite of this, China's certification system is still weak, without the face-to-face teaching and assessment in reality, how to evaluate students' daily performance? How do you guarantee that the students who get the credits are the ones who are studying in the course? How to prevent the phenomenon of cheating and seeking surrogate exam-taker among students to guarantee the high quality of credit? All these problems require further exploration and research.

\section{EFFective Path to Complete Educational PATTERN OF MOOC}

As a new mode of education, MOOC will deeply influence the future of further education, how to overcome the challenges in the process of development is the study of the domestic "MOOC" model systematic problems in sustainable development.

\section{A. Building a unified MOOC platform and multi-channel publicity and promotion.}

After several years of development, MOOC in western developed countries has begun to take shape. The three major MOOC providers "Coursera", "Udacity" and "Edx" have taken the lead. Our local MOOC platforms can also learn from them and establish a unified MOOC platform to break the barriers between colleges and universities, strengthen cooperation in a unified MOOC platform, increasing the technical talent cultivation and introduction, and build a strong professional technical support team which is responsible for the day-to-day operation and maintenance of the MOOC platform and pushes courses on a regular basis, timely solves sudden problems of the platform. In addition, with a unified platform as a technical support, the course production team can focus on the selection and design of the courses' content. What's more, for enrolled users, a unified platform integrates multiple resources, which makes it easier to retrieve information, to select courses that interest them, to learn the knowledge needed, and to improve learning efficiency.

When it comes to the promotion of MOOC platform, on one hand, it can not only establish contact with traditional news media publicity media, but also make MOOC no longer distant and unknown. On the other hand, we have reached cooperation with online social platforms such as Weibo and WeChat to promote the promotion of new courses and enhance the interaction with users. Through multi-channel promotion, longed MOOC audience not only confined to the college students, white-collar work, but as a means of education, helping people spend leisure time and improve their own quality of emerging education way.

\section{B. Improving the quality of the courses in many ways}

The creation of the MOOC platform is to provide quality courses for more people. The quality of courses is the core of MOOCs. A good course should attract more enrolled users. The quality of the courses can be improved from two aspects. On one hand, improve the professional quality of lecturers. The professional competence of lecturers directly influences enrolled users' learning interest and enthusiasm. Teachers should improve their own teaching ability, enrich their teaching art, change the teaching concept, define their role orientation, change from a speaker to the guide, and adapt to the new education mode. On the other hand, the course production team deeply understands the connotation of MOOC and provides scientific and reasonable curriculum design for the keynote speaker. The course of MOOC is intended to make full use of the time of enrolled users' fragments, to carry out in-depth explanation of the knowledge points, so that enrolled users can be highly concentrated on a short period of time, so as to achieve proficiency in knowledge. 
Therefore, the course production team should focus on building the curriculum learning system and controlling video time.

\section{Making a series of courses with their own characteristics}

The continuous development of the new education mode has become an important means to disseminate the excellent culture and education resources of various countries. We can pursue the doctrine of "bring doctrine" to the existing highquality MOOC courses abroad. Organize the team to work on the system and subtitle production to reduce duplication of labor. And our local MOOC in addition to joining the international MOOC platform, should pay more attention to the development of courses which have its own characteristics and which can show Chinese rich culture and history to foreigners.

\section{Broadening financing channels and exploring commercial operation mode}

On one hand, the government appropriately increased the financial allocation of education project to establish an independent fund project for the development of MOOC. Colleges and universities, on the other hand, can be sponsored by recruiting, and can attract alumni donations or cooperate with the third party company to develop registered qualification certification courses, vocational and technical training or professional degree, reduce the burden of financing. The commercial operation of MOOCs seems to be contrary to the original intention of large-scale opening, but it is not enough to support the sustainable development of the MOOC platform by solely relying on the joint investment of government and universities. By introducing financial capital and exploring the commercial operation of MOOCs, the MOOC platform can be self-sufficient, and the profits can be put into the future development of the platform. By referring to the more mature profit experience of foreign platforms, the MOOC platform can also choose to raise funds by granting certificates, advertising in the platforms and providing personalized service for specific people [6].

\section{E. Establishing a sound certification system.}

At present, the domestic credit certification system is not perfect, and the certification is not strong. Some colleges and universities admit the credit of the MOOCs, but it is still not unified standard in the cross-school curriculum. It is imperative to actively construct the credit mutual recognition system among colleges and universities, sharing high-quality courses and reducing the gap of education resources. Domestic colleges and universities can also combine their own characteristics, learning from foreign credit certification mechanism, to establish standard credit certification and establish their own credit certification mechanism. In addition, although some of the MOOC enrolled users are registered for their own interest, most of them study MOOC for the purpose of adding personal skills to help them in their future work and life. In order to make the MOOCs certificate also have the certification effect in the enterprise, we should increase the gold content of the certification, which can stabilize the source and improve the learning initiative. The MOOC platform should actively establish contacts and seek cooperation with external enterprises.

\section{CONCLUSION}

The emergence and vigorous development of MOOCs have impacted the traditional education mode, but it also provides new ideas for people to use education resources equally and promotes the promotion and popularization of education. Although the development of MOOCs encounter some problems at the present stage, the community is still trying to find a solution. The future development of MOOCs should actively overcome its shortcomings, giving full play to its technical advantages, and focus on meeting the personalized needs of various parties. We believe that MOOCs will seize opportunities and ushers in a broader development prospect.

\section{REFERENCES}

[1] M ahnaz M oallem, “An Interactive Online Course: A Collaborative Design Model” [EB/OL]. Educational Technology Research and Development,2003, (4)

[2] Li Shuang, Gu Liang-ling, "Thinking About the Revolution of MOOC," [J]. Contemporary Education Research and Teaching, 2016, (10), In Chinese

[3] Wei Zhi-min, "The Problems and Countermeasures of MOOC Localization Development.” [J]. Journal of Northwest Normal University, 2015, (1):80, In Chinese

[4] Ding Wan-yi, “Analysis and Suggestions on the Bottleneck of MOOC Development in China," [J]. Journal of the Open University of Guangdong, 2016, (1):4., In Chinese

[5] Cai Zhong-bing, Luo Zhi-wen, "The construction of colleges MOOC: current situation, problems and trends," [J] Higher Education Exploration, 2017, (11):48-49, In Chinese

[6] Zhai Yu-hui, Yang Ming-hui., "Development Status and Problems Research of MOOC in China," [J]. Journal of Hubei University of Science and Technology, 2016, (5):96-97, In Chinese 